Cahiers
de a $\begin{gathered}\text { Recherche } \\ \text { sur les Droits }\end{gathered}$

Cahiers de la recherche sur les droits

Fondamentaux fondamentaux

16 | 2018

Les partis politiques

\title{
Le rôle des partis politiques en Tunisie : entre quête du pouvoir et pacification sociale
}

The Role of Political Parties in Tunisia: Between Search for Power and Social

Pacification

Wafa Tamzini

\section{CpenEdition}

Journals

Édition électronique

URL : https://journals.openedition.org/crdf/311

DOI : $10.4000 /$ crdf.311

ISSN : 2264-1246

Éditeur

Presses universitaires de Caen

Édition imprimée

Date de publication : 16 novembre 2018

Pagination : 69-78

ISBN : 978-2-84133-901-3

ISSN : 1634-8842

Référence électronique

Wafa Tamzini, «Le rôle des partis politiques en Tunisie : entre quête du pouvoir et pacification

sociale », Cahiers de la recherche sur les droits fondamentaux [En ligne], 16 | 2018, mis en ligne le 16

novembre 2019, consulté le 14 novembre 2022. URL : http://journals.openedition.org/crdf/311 ; DOI :

https://doi.org/10.4000/crdf.311 


\title{
Le rôle des partis politiques en Tunisie: entre quête du pouvoir et pacification sociale
}

\author{
Wafa TAMZINI \\ Maître de conférences en droit public à l'université Sorbonne Paris Cité \\ Directrice de I'Institut de recherches sur les mondes méditerranéen et africain (IRMMA) \\ Centre d'études et de recherches administratives et politiques (CERAP, EA 1629) \\ Membre associée du Centre d'études et de recherches de sciences administratives et politiques (CERSA, UMR 7106)
}

I. Le rôle institutionnel des partis politiques
A. De la création d'un apparent multipartisme...
B. ...à la mise en place d'un véritable monopartisme

II. Le rôle social des partis politiques

A. Le Rassemblement démocratique constitutionnel: entre verrouillage institutionnel et médiation sociale

B. Le paradoxe de la révolution de jasmin: entre un pluripartisme effectif et la multiplication des mouvements sociaux à l'extérieur du champ partisan

Les pays arabes ont en commun une histoire, une langue et une culture influencées par l'islam. Depuis le soulèvement populaire de décembre 2010 en Tunisie et ce que l'on nomme communément le "printemps arabe», l'ensemble des peuples arabes aspire à davantage de liberté. Bien que partageant un certain nombre de traits communs, il n'en demeure pas moins que chacun de ces États possède sa propre dynamique, son propre rythme d'évolution. C'est la raison pour laquelle les soulèvements populaires qui se sont déroulés dans cette aire géographique n'ont pas tous eu les mêmes conséquences juridiques.

En Tunisie, la révolution "de jasmin », débutée le 17 décembre 2010 après l'immolation du jeune Bouazizi, a abouti au terme d'une transition constitutionnelle à l'adoption le 26 janvier 2014 d'une nouvelle Constitution. En Égypte, la chute du régime de Hosni Moubarak a conduit à l'adoption d'une nouvelle Constitution le 30 mars 2011 qui sera modifiée en décembre 2012 sous le règne de Mohamed Morsi, président nouvellement élu, puis remplacée les 14 et 15 janvier 2014 par une nouvelle norme fondamentale, après la destitution par l'armée du chef de l'État.

Formellement, la Tunisie et l'Égypte sont les seuls pays de ce "printemps arabe» à avoir adopté une nouvelle Constitution. Au Maroc, le pouvoir en place a en effet devancé les aspirations démocratiques des citoyens dans le but d'éviter de vivre un scénario tunisien. Une réforme constitutionnelle a bien eu lieu, même si elle fut critiquée par l'opposition, notamment au cours du mouvement du 20 février $2011^{1}$. Sans minimiser les avancées introduites par le pouvoir constituant, celles-ci n'ont pas été associées à de véritables changements structurels notamment dans la protection des droits et libertés. En revanche, concernant l'Algérie, aucune modification constitutionnelle n'a été entreprise, malgré les promesses répétées du chef de l'État. Et il en va de même, pour l'heure, en Libye. Si une commission spéciale fut chargée, le 21 avril 2014, d'élaborer une nouvelle Constitution avec pour contrainte temporelle

1. Voir O. Bendourou, «La nouvelle Constitution marocaine du 29 juillet 2011 », Revue française de droit constitutionnel, $\mathrm{n}^{\circ}$ 91, $2012, \mathrm{p} .511-535$. 
d'achever cette mission dans un délai de cent vingt jours, les tensions politiques ont eu raison de cet objectif ${ }^{2}$. En Irak, de même qu'au Yémen, c'est la persistance d'une forte instabilité politique et militaire qui empêche (encore) la mise en œuvre d'une transition constitutionnelle.

Ces périodes politiques, caractérisées par des troubles juridiques, des vacillements constitutionnels et des errements institutionnels, sont complexifiées par la volonté des rédacteurs d'élaborer de nouvelles Constitutions en s'efforçant dans la mesure du possible de faire table rase du passé ${ }^{3}$. En Tunisie, précisément, l'Assemblée nationale constituante aurait pu décider de conserver l'ancien texte de 1959 et d'effectuer uniquement un travail de révision. Les députés de l'Assemblée nationale constituante ont néanmoins privilégié la rédaction d'un nouveau texte, avec cette volonté, largement encouragée par la société civile, de faire disparaître tant les stigmates du régime déchu ${ }^{4}$ que les traces historiques des multiples et diverses occupations coloniales, dont, en dernier lieu, celle de l'État français.

Après le soulèvement populaire ayant conduit à la fuite du président, les dirigeants politiques tunisiens encore au pouvoir avaient hésité entre deux interprétations possibles de la Constitution. Soit les articles 56 et 57 de l'ancien texte trouvaient à s'appliquer et le président déchu devait être remplacé comme le prévoient ces textes; soit aucune disposition constitutionnelle n'avait vocation à être mise en œuvre et, dans cette hypothèse, la décision de remplacer l'ancien texte par une nouvelle Constitution s'imposait.

La solution adoptée fut toutefois d'appliquer les dispositions constitutionnelles alors toujours en vigueur. L'article 56 de la Constitution de 1959 disposait qu'en

[...] cas d'empêchement provisoire, le Président de la République [pouvait] déléguer par décret ses attributions au Premier ministre, à l'exclusion du pouvoir de dissolution de la Chambre des députés.

En application de ces dispositions, l'ancien Premier ministre, Rached Ghannouchi, avait décidé de remplacer le président déchu. Cette décision n'avait pas réussi à calmer la colère des manifestants qui ne voulaient pas se contenter de ce remplacement, même temporaire. D'autant que, d'un point de vue juridique, l'application de cet article ne se justifiait pas, le chef de l'État n'ayant, avant sa fuite, rédigé aucun décret de délégation de pouvoirs. L'article 57 avait ensuite été mis en application par le pouvoir exécutif. Ce dernier portait sur la vacance de la présidence de la République et prévoyait que dans l'hypothèse d'un décès, d'une démission ou d'un empêchement absolu du président de la République, il appartenait au Conseil constitutionnel de se réunir immédiatement et de constater la vacance définitive à la majorité absolue de ses membres. C'est ainsi que, le 15 janvier 2011, la juridiction suprême se réunit et constata la vacance du pouvoir, conformément aux dispositions constitutionnelles. Après cette déclaration de vacance du pouvoir, il revenait au président de la Chambre des députés d'assurer les fonctions de président de la République par intérim. Là encore, le texte avait été respecté et le président de la Chambre des députés était devenu à son tour et provisoirement président de la République. C'est d'ailleurs sous sa présidence qu'ont été adoptés un certain nombre de textes censés organiser la transition, dont le décret-loi $\mathrm{n}^{\circ}$ 2011-6 du 18 février 2011 portant organisation provisoire des pouvoirs publics et créant une instance supérieure pour la réalisation des objectifs de la révolution, de la réforme politique et de la transition démocratique. Cette instance n'avait pas été investie de la mission de rédiger une nouvelle Constitution. Elle était chargée, conformément aux dispositions de l'article 2 dudit décret-loi,

[...] d'étudier les textes législatifs ayant trait à l'organisation politique et de proposer les réformes à même de concrétiser les objectifs de la révolution relatifs au processus démocratique.

Le type de processus constituant n'était donc pas encore celui que l'on connaîtra finalement. La Tunisie s'orientait bien initialement vers une modification de la Constitution de 1959 et non vers la rédaction d'un nouveau texte. Ce n'est qu'en mars 2011 que la décision fut prise par le pouvoir exécutif d'organiser des élections en vue de mettre en place une Assemblée nationale constituante dont la mission consistera à élaborer l'actuelle Constitution du 26 janvier 2014. Mais, entre-temps, et sur le fondement du décret-loi n ${ }^{\circ}$ 2011-6 du 18 février 2011, ont été adoptées des dispositions relatives à la Constitution et au financement des partis politiques. Il s'agit du décret-loi $\mathrm{n}^{\circ}$ 2011-87 du 24 septembre 2011 portant organisation des partis politiques.

2. La commission devait théoriquement être composée de soixante membres. Or, treize d'entre eux n'ont pas pu être élus en raison de l'instabilité sécuritaire présente dans leur région électorale.

3. C'est en se fondant sur la théorie de la "page blanche» que les pouvoirs constituants considèrent fréquemment devoir mener à bien leur travail de rédaction. Cette théorie connaît cependant des limites factuelles en raison, d'une part, de l'impossibilité de nier entièrement un passé juridique, une mémoire constitutionnelle et, d'autre part, parce que les termes désignant les institutions sont immuables (un président de la République, un Premier ministre, etc.).

4. La volonté de vouloir faire table rase du passé intervient fréquemment lors d'une révolution. Ainsi, en 1944, après la libération du territoire national, De Gaulle n'avait-il pas décidé, par une ordonnance qui a fait date, de déclarer nuls tous les actes juridiques (constitutionnels, législatifs ou réglementaires) promulgués après le 16 juin 1940, dans le but de «libérer le pays de la réglementation d'inspiration ennemie qui l'étouffait » (ordonnance du 9 août 1944 relative au rétablissement de la légalité républicaine sur le territoire continental)? Cette déclaration, portant disparition juridique et rétroactive des actes du gouvernement de Vichy, rencontrait des limites; de nombreux textes survécurent à cette annulation symbolique ( «dans les faits, des actes de Vichy ont été validés provisoirement ou définitivement, annulés partiellement ou totalement, des actes dits lois de Vichy ou encore lois validées sont visés en début d'ordonnance»). Il s'agissait bien pour De Gaulle de trouver dans «cette annulation un moyen d'asseoir sa propre légitimité et de fixer ainsi sa propre compétence» (W. Tamzini, «Vichy: un passé qui ne passe décidément pas...», in Voyages en l'honneur du professeur Geneviève Koubi, M. Touzeil-Divina, G. Guglielmi (dir.), Le Mans, Éditions l'Épitoge, 2012, p. 219-228). 
C'est ce texte qui régit encore le cadre juridique des partis en Tunisie, qui peuvent être définis comme

[...] des groupements volontaires plus ou moins organisés, qui prétendent, au nom d'une certaine conception de l'intérêt commun et de la société, assurer seuls ou en coalition, les fonctions de gouvernement ${ }^{5}$.

L'article 2 dudit décret-loi donne une définition similaire puisque le parti politique y est défini comme étant

[...] une association constituée sur la base d'un accord entre citoyens tunisiens, qui contribue à l'encadrement politique des citoyens et à la consécration des valeurs de la citoyenneté. Il a pour objectif la participation aux élections en vue d'exercer le pouvoir au niveau national, régional ou local ${ }^{6}$.

Jusqu’à la révolution de décembre 2010, il n'existait essentiellement qu'un parti politique dominant l'ensemble de la vie politique. Depuis le changement de régime, le nombre des partis politiques en Tunisie dépasse allègrement la centaine pour atteindre deux cent dix le 22 novembre 2017 avec la création par Ahmed Nejib Chebbi ${ }^{7} \mathrm{du}$ « mouvement démocrate». Cette profusion de partis politiques conduit actuellement les autorités gouvernementales à préparer un projet de loi organique régissant les partis politiques pour la fin du premier semestre 2018. Le chef de cabinet du ministre des relations avec les Instances constitutionnelles, la Société civile et les Droits de l'homme, Mondher Bousnina a précisé qu'il s'agissait de concilier les dispositions constitutionnelles actuelles (notamment l'article 65 de la Constitution) avec l'esprit du décret-loi $\mathrm{n}^{\circ}$ 2011-87 portant organisation des partis politiques. En effet, il convient selon lui

[...] d'aligner la loi sur les partis politiques aux standards internationaux et d'éclaircir certains points dont notamment la question du financement public qui se limite aujourd'hui au financement des campagnes électorales ${ }^{8}$.

La multiplication des partis politiques depuis 2011 contraste avec les régimes politiques précédents caractérisés par l'existence d'une formation prédominante et de quelques partis d'opposition récemment tolérés. Précisément,

L'image la plus partagée sur la situation politique de ce petit pays d'un peu plus de 9 millions d'habitants, qui a pour voisins directs l'Algérie et la Libye, est aujourd'hui celle de l'inexistence d'un marché politique concurrentiel: s'il existe des partis politiques, et un espace légal d'activité politique partisane, ils ne jouent d'aucune façon le rôle d'opposition politique au régime en place ${ }^{9}$.
Partant, même si la Tunisie est le premier pays arabe à se doter d'une Constitution dès 1861, il faudra attendre l'adoption du décret du 29 décembre 1955 pour voir apparaitre une vie parlementaire avec l'institution d'une Assemblée constituante chargée de rédiger la future norme fondamentale post-indépendance. Malgré la création d'un Parlement en 1959, la vie politique restera marquée par la puissance du Parti destourien sous le régime de Bourguiba, puis de celle du Rassemblement constitutionnel démocratique sous le régime de Ben Ali.

Depuis la révolution de jasmin, deux grandes formations dominent la vie politique tunisienne: il s'agit d'une part de Nidaa Tounes (Appel de la Tunisie) qui est un parti politique tunisien créé par l'actuel président de la République - Béji Caïd Essebsi - le 6 juillet 2012, et d'autre part du mouvement Ennahda (Mouvement de la renaissance) qui est un parti créé le 6 juin 1981 sous le nom de Mouvement de la tendance islamique. Les résultats des dernières élections législatives du 26 octobre 2014 ont placé Nidaa Tounes en tête des suffrages mais sans majorité absolue avec l'obtention de 86 sièges sur les 217 sièges que compte la nouvelle Assemblée des représentants du peuple, suivi du mouvement Ennahda qui se place en deuxième position avec 69 sièges. Plus d'une dizaine de partis politiques se partagent les 62 sièges restants.

La multiplication des partis politiques atteste d'une réelle démocratisation de la vie politique. Pour surmonter les difficultés liées à la profusion des formations politiques, certains partis ont décidé de s'unir et de présenter des listes communes aux premières élections locales libres afin de clarifier pour l'électeur l'offre politique soumise à son appréciation. S'il est indéniable que les partis politiques dans la Tunisie post-révolutionnaire assument une fonction institutionnelle réelle, il n'en demeure pas moins que ceux-ci peinent à assurer leur fonction sociale comme en attestent les multiples mouvements de protestation issus de la société civile ces dernières années. En effet, dès 2011, de nombreux citoyens ont créé des mouvements de contestation, de revendication et / ou de contrôle de l'action publique en dehors du champ politique. Il en est ainsi, par exemple, du mouvement citoyen Al Bawsala (La boussole) créé en 2011. Il s'agit d'une organisation non gouvernementale qui poursuit plusieurs objectifs, notamment celui visant à informer les citoyens des activités de leurs élus afin de promouvoir la transparence et l'éthique politique. De même, des citoyens ont presque spontanément créé un mouvement intitulé Mouch Bessif («Pas contre notre volonté») pour

5. R. Aron, Démocratie et totalitarisme, Paris, Gallimard, 1965, p. 117.

6. Art. 2 du décret-loi $\mathrm{n}^{\circ} 2011-87$ du 24 septembre 2011 portant organisation des partis politiques, Journal officiel de la République tunisienne, $\mathrm{n}^{\circ} 74$, p. 1973 .

7. Ancien fondateur du Parti démocrate progressiste (PDP) devenu un temps le parti El Jomhouri.

8. «Création des partis politiques. Vers l'harmonisation des législations», La presse de Tunisie, 29 novembre 2017, en ligne: http://www.lapresse.tn/ component/nationals/?task=article\&id $=140080$

9. C. Braun, "À quoi servent les partis tunisiens? Sens et contre-sens d'une "libéralisation" politique", Revue des mondes musulmans et de la Méditerranée, $\mathrm{n}^{\circ}$ 111-112, mars 2006, p. 16 
dénoncer la pression exercée sur les non-jeûneurs durant la période de Ramadan. Ils ont organisé la première manifestation à Tunis le 11 juin 2017 pour exiger le respect de la liberté de conscience qui est désormais consacrée au niveau constitutionnel ${ }^{10}$.

Les partis politiques en Tunisie occupent une place institutionnelle importante depuis longtemps en Tunisie. Mais ce n'est que très récemment que les formations partisanes sont libres de se constituer et ne sont plus simplement réduites au rôle de spectateur par le pouvoir en place comme ce fut le cas sous les deux régimes autoritaires qui se succédèrent de 1956 à 2010 (I). Pour autant, la fin du monopartisme et l'avènement du pluralisme politique n'ont pas encore su faire oublier les stigmates des anciens régimes et la méfiance des citoyens vis-àvis de ces multiples formations, incitant ces derniers à préférer se regrouper et à agir en dehors de la sphère strictement politique pour exprimer des revendications sociales (II).

\section{Le rôle institutionnel des partis politiques}

Dans l'histoire récente de la Tunisie, les partis politiques occupent une fonction institutionnelle importante. Il faut remonter à la période du protectorat pour situer l'apparition de partis politiques. À l'époque, les différentes formations s'entendaient pour lutter contre la colonisation. Une fois l'indépendance acquise, le multipartisme a vite laissé place au monopartisme incarné par le Parti destourien, formation dirigée par Habib Bourguiba. Cette hégémonie d'un parti unique a persisté sous le régime de Zine el-Abidine Ben Ali avec le Rassemblement constitutionnel démocratique. La chute du régime en 2011 a laissé place à une libéralisation du champ politique qui a suscité l'éclosion de nouveaux partis politiques.

\section{A. De la création d'un apparent multipartisme...}

Après l'indépendance, le système partisan en Tunisie se caractérisait par l'hégémonie d'une grande formation politique: le parti libéral Néo-Destour qui deviendra par la suite le Parti socialiste tunisien. Celui-ci est le successeur du parti du vieux Destour ${ }^{11}$ créé en 1920 autour d'une élite principalement bourgeoise et qui revendique la création d'une Constitution accompagnée de réformes socio-économiques et institutionnelles. Les membres de ce parti, dirigé par l'un de ses fondateurs le cheikh Abdelaziz Thâalbi, rédigent un programme comportant notamment la création d'un Parlement élu ou encore l'instauration de l'indépendance du pouvoir judiciaire ${ }^{12}$.

Certains membres de ce parti réclamaient davantage que de simples réformes et s'opposaient ainsi aux plus anciens, leur reprochant de ne pas réclamer purement et simplement l'indépendance du pays. Parmi ces jeunes «rebelles» figurait Habib Bourguiba, l'un des futurs leaders de la lutte anticoloniale. Ceux-ci vont se regrouper et créer le Néo-Destour le 2 mars $1934^{13}$. Le pouvoir est réparti entre plusieurs personnes au sein du parti et « [s]i le jeune et brillant avocat et journaliste Habib Bourguiba en est le leader, le pouvoir est à l'origine distribué au sein d'un cercle assez large ${ }^{14}$. Mais cette répartition est davantage due au contexte qu'à une réelle volonté de partage du pouvoir puisque les «dix années que Bourguiba passe en prison entre 1934 et 1955, et la répression dont fait l'objet le Néo-Destour à cette époque, forcent à cette distribution non restreinte du pouvoir au sein du parti $»^{15}$.

C'est la première organisation politique qui s'adresse à tous les Tunisiens quelle que soit leur origine socioprofessionnelle ou même géographique ${ }^{16}$. Mais cette situation ne persistera pas après l'indépendance. Lors des négociations sur le sort de la Tunisie qui eurent lieu entre les autorités françaises et les dirigeants du

10. «L'État est gardien de la religion. Il garantit la liberté de croyance, de conscience et le libre exercice des cultes [...]» (art. 6 de la Constitution tunisienne).

11. «Destour est un mot d'origine perse et signifie constitution (le terme arabe moderne est "dustur"). Adossé à l'expression de parti politique, le mot Destour renvoie à l'idée d'une ambition nationaliste, visant à établir une nouvelle Constitution et dont le but principal est l'obtention de l'indépendance de la Tunisie. Successeur du mouvement des Jeunes Tunisiens, le Destour ou de son nom officiel "le parti libéral constitutionnel tunisien" naît en 1920 et attire rapidement l'adhésion de nombreux notables et intellectuels soucieux de libérer la Tunisie du protectorat français. Le Destour se scindera en deux en 1934; le Néo-Destour verra le jour et sera finalement dirigé par Habib Bourguiba. Le Néo-Destour est plus vindicatif que le Destour et réclame l'indépendance de la Tunisie, ne se contentant plus de simples concessions de la part de l'autorité coloniale. Ce parti disparaîtra en 1964 pour laisser place au parti socialiste destourien» (W. Tamzini, La Tunisie, Bruxelles, De Boeck, 2013, p. 12).

12. Voir Dustur: aperçu sur les constitutions des États arabes et islamiques, Leyde, E. J. Brill, 1966, p. 1-4.

13. «[...] dès 1933, ce jeune intellectuel décide d'orienter le Destour dans un sens moins bourgeois et plus tunisien, laïque et libéral. L'année suivante, le divorce entre le Destour et le mouvement d'Habib Bourguiba, le Néo-Destour, est consommé. Les dirigeants du Néo-Destour sont pourchassés et déportés dans le Sud. Il faudra attendre 1936 et le gouvernement de Léon Blum pour assister à leur libération. Des négociations s'engagent entre les autorités politiques tunisiennes et françaises, mais sont stoppées avec la chute du gouvernement français en 1937. S'ensuivent des émeutes sanglantes et de nouveau l'arrestation des chefs du Néo-Destour et la proclamation de l'état de siège à compter de 1938 " (W. Tamzini, La Tunisie, p. 12-13)

14. C. Braun, «À quoi servent les partis tunisiens?...», p. 19.

15. Ibid.

16. Ainsi que le montre Céline Braun: «En mars 1934, le Néo-Destour est fondé et rejette la vieille garde de l’ancien Destour, représentée intellectuellement par un cheikh de la Zitouna, Abdelaziz Thaalbi, auquel s'oppose violemment la jeune génération de militants. La majorité du soutien du parti provient déjà des villes de la côte et des villages du Sahel plutôt que de la capitale, de ses vieilles familles et de ses oulémas. Ainsi, le village de Ksar Hellal fut le lieu du premier congrès du Néo-Destour en 1934. L'élite provinciale deviendra le principal élément de soutien au Néo-Destour, mais le parti ralliera aussi progressivement les éléments traditionnels et appauvris de la capitale que la faction plus dure représentée par Salah Ben Youssef avait dans un premier temps attirés. Le Néo-Destour est donc la première organisation politique à s'ouvrir aux Tunisiens de toutes les régions et de tous les groupes sociaux» (ibid., p. 19). 
Néo-Destour, une forte opposition eut lieu entre les deux figures majeures du parti: Habib Bourguiba et Salah Ben Youssef. Ce dernier paraissait trop excessif dans ses revendications aux yeux des Français, lesquels préférèrent soutenir Habib Bourguiba qui prônait une approche plus modérée. Partant, les militants qui soutenaient Salah Ben Youssef commencèrent à s'opposer en interne à l'aile bourguibienne du parti. Cette manifestation de divergence quant aux modalités de l'acquisition de l'indépendance a conduit à une sévère répression des yousséfistes et leur leader fut contraint à s'exiler en Lybie dès 1956 avant d'être assassiné en Allemagne quelques années plus tard. Il n'y avait donc plus d'opposition interne au Néo-Destour, permettant ainsi à Habib Bourguiba de prendre entièrement le pouvoir du parti. L'exercice monopolistique du pouvoir exercé personnellement par le futur premier président de la République tunisienne ne cessera plus jusqu'à sa destitution en 1987. Pourtant, la constitution du parti du Néo-Destour s'apparentait fortement à celle d'un parti politique français, construit sur un modèle certes centralisé mais qui se voulait, du moins en apparence, démocratique. En effet, c'est le «principe du centralisme démocratique» qui «régit théoriquement le fonctionnement du parti $»^{17}$. Mais la personnalisation du pouvoir par son leader empêchera tout exercice démocratique de la gestion du mouvement. Et cela d'autant plus qu'une fois élu au sommet de l'État, Habib Bourguiba personnalisera également l'exercice de ses compétences et amorcera ainsi la confusion entre État et parti.

En 1964, le Néo-Destour devient le Parti socialiste destourien et scelle définitivement son incapacité à mettre en place un exercice démocratique du pouvoir, en ne tolérant aucune opposition politique et en plaçant ses membres à tous les postes et fonctions importants du nouvel État alors en construction. Les articles constitutionnels prédisposent le régime politique à une dérive autoritaire puisque la Constitution du $1^{\text {er }}$ janvier 1959 mettra en place un régime présidentiel fort ${ }^{18}$ et une Assemblée qui s'avérera faible dans la mesure où cette dernière sera en réalité réduite au plus petit rôle par l'usage par le chef de l'État de ses prérogatives. La Constitution prévoyait pourtant dans son article 8 la liberté d'expression ainsi que la liberté d'association. Mais ces dispositions ont rapidement été vidées de leur substance par l'entrée en vigueur de la loi n ${ }^{\circ}$ 59-154 du 7 novembre 1959 relative aux associations ${ }^{19}$ qui apportait des restrictions à l'exercice de cette liberté. À titre d'illustration, l'article 4 de ladite loi prévoyait qu'une association ne pouvait «légalement exister qu'après visa de ses statuts par le secrétaire d'État à l'Intérieur ${ }^{20}$. De même, on aurait pu nourrir l'espoir d'une réelle démocratisation du système partisan avec la tenue des premières élections législatives post-indépendance de Tunisie. Mais, là encore, ce ne fut pas le cas. En effet, les premières élections législatives du régime eurent lieu en 1959 et - par une habile stratégie électorale $^{21}$ - donnèrent lieu à la victoire des destouriens qui formeront alors une Assemblée docile, voire même servile, puisque cette dernière ne se réunira sous Habib Bourguiba «que pour adopter les législations élaborées par celui-ci, qui passe d'ailleurs au-dessus de l'Assemblée en émettant des décrets pendant les six mois de l'année où l'Assemblée ne se réunit pas ${ }^{22}$. Précisons que les dispositions constitutionnelles permettent cette situation dans la mesure où l'article 29 dispose que l' «Assemblée nationale se réunit en deux sessions extraordinaires par an, dont la durée, déterminée par la loi, ne peut excéder trois mois pour chacune d'elles». De plus, l'article 31 énonce que:

Le président de la République peut, pendant les vacances de l'Assemblée, prendre, avec l'accord de la commission permanente intéressée, des décrets lois qui doivent être soumis à la ratification de l'Assemblée au cours de la session ordinaire suivante.

L'exercice d'un pouvoir personnel par Habib Bourguiba atteint son paroxysme après la réforme du parti de 1958. Cette réforme sera complétée par l'instauration de la règle du parti unique en 1963. Cette institutionnalisation du parti unique s'explique par la volonté du chef du parti de museler toute opposition pour éviter à l'avenir ce qui s'est produit en 1962 avec la tentative de coup d'État fomenté par des citoyens yousséfistes. Désormais, le système est verrouillé et toute opposition institutionnelle est rendue impossible. Ce d'autant que le président de la République - qui est également chef du parti unique et qui se présente comme étant le «père de la nation $»^{23}$ - est désormais élu à vie depuis la révision constitutionnelle intervenue en 1974.

17. C. Braun, «À quoi servent les partis tunisiens?...», p. 2o. L'auteure décrit ainsi le système existant sous le protectorat et censé permettre l'exercice démocratique du pouvoir au sein du Néo-Destour : «le Congrès national, composé des délégués de toutes les cellules et autorité suprême du parti, élit le Bureau politique qui donne à son tour des directives aux fédérations, qui les font elles-mêmes redescendre aux cellules. Sous le protectorat, le fonctionnement théorique du parti n'est pas véritablement appliqué et les Bureaux politiques se réunissent dans la clandestinité » (ibid.).

18. L'article 28 dispose ainsi que l'Assemblée exerce le pouvoir législatif mais que l'initiative des lois «appartient concurremment au président de la République et aux membres de l'Assemblée, les projets présentés par le président de la République ayant la priorité».

19. Loi nº 59-154 du 7 novembre 1959 relative aux associations, Journal officiel de la République tunisienne, $\mathrm{n}^{\circ}$ 63, 22 décembre $1959, \mathrm{p} .1534$.

20. Ibid.

21. Le bey promulgue une loi électorale en janvier 1956 sous la pression de Bourguiba. Cette loi mettra en place un scrutin de liste majoritaire à un tour «qui garantit la domination du Parti destourien en éliminant les petits partis et les opposants indépendants» (C. Braun, «À quoi servent les partis tunisiens?...», p. 21).

22. C. Braun, "À quoi servent les partis tunisiens?... ", p. 21-22.

23. Ibid., p. 22 


\section{B. ...à la mise en place d'un véritable monopartisme}

Ce monolithisme partisan ainsi institutionnalisé durera jusqu'à la destitution du président de la République, également chef du parti du Néo-Destour, lequel a été rebaptisé entre-temps Parti socialiste destourien. En effet, malgré l'apparente démocratisation intervenue en 1981 avec l'introduction du pluralisme lors des élections législatives de la même année, le Parti socialiste destourien remporte les élections et aucun membre de l'opposition ne parvient à se faire élire. Dans le même temps, l'Union générale des travailleurs tunisiens qui pouvait s'apparenter à de l'opposition non partisane et qui aurait pu ainsi jouer le rôle de catalyseur des revendications et mécontentements populaires est également aux mains de membres appartenant au parti unique. Partant, excepté le Parti communiste qui a été créé en 1920 et qui sera d'ailleurs le premier parti politique d'opposition à être légalisé en 1981, tous les mouvements partisans qui tentent de percer sur le plan électoral sont issus du Parti socialiste destourien. Il en va ainsi du Mouvement des démocrates socialistes, fondé en 1978 par Ahmed Mestiri, membre historique du parti néodestourien, ancien ministre pendant les années 1960 et qui réclamait davantage de démocratie dans la vie politique. De même, le Mouvement de l'unité populaire sera créé par un ancien compagnon de lutte de Habib Bourguiba mais qui deviendra par la suite son opposant, Ahmed Ben Salah, lequel sera emprisonné mais réussira à s'enfuir en 1973 et créera depuis l'étranger ce mouvement d'opposition au régime bourguibien. Ces deux mouvements seront légalisés en 1983 et se verront donc intégrés au système partisan officiel. Ces légalisations sont des moyens pour le pouvoir en place de contrôler toutes les formes d'opposition.

Le même sort est réservé aux organisations non partisanes. Ainsi, l'Union générale des travailleurs tunisiens ${ }^{24}$ était à l'origine aux côtés du parti du Néo-Destour durant les années 1950. Cette organisation syndicale a par la suite été associée étroitement à l'exercice du pouvoir en la personne de son dirigeant, Ahmed Ben Salah, qui a été également ministre de l'Économie et a dirigé l'expérience collectiviste durant cinq années. Mais après l'échec de cette politique économique, l'Union générale des travailleurs tunisiens redevient une force syndicale en soutenant, notamment, les mouvements de grève des année 1970. C'est ainsi que de partenaire cette organisation deviendra un ennemi à abattre pour le pouvoir en place. D'ailleurs, plusieurs centaines de syndicalistes seront arrêtées et contraindront l'Union générale des travailleurs tunisiens à redevenir obéissante.

Il en est également ainsi du Mouvement de la tendance islamique qui voit le jour en Tunisie à la fin des années 1960 avec pour leitmotiv principal le refus d'une société sécularisée. Leur leader Rached Ghannouchi - qui est aujourd'hui une figure incontournable du paysage politique - avait compris très rapidement le profit que son mouvement pouvait tirer des manifestations sociales et syndicales. Progressivement, le Mouvement de la tendance islamique s'intéresse à la lutte pour le pouvoir et décide d'y prendre part, lorsque Habib Bourguiba accepte en 1981 que plusieurs partis politiques participent pour la première fois à des élections, à charge pour eux de déposer une demande de légalisation la même année. Mais la réponse du pouvoir fut violente puisqu'en juillet 1981 « une soixantaine de membres du Mouvement de la tendance islamique - dont Ghannouchi - sont arrêtés et jugés pour formation d'organisation illégale, diffamation du président et diffusion de fausses nouvelles ${ }^{25}$. Malgré ces arrestations, le mouvement reste actif et influent dans la société, conduisant le Combattant suprême ${ }^{26}$ à accepter de les amnistier en échange de l'abandon de l'usage de la violence par le Mouvement de la tendance islamique. Mais les islamistes ont continué de participer activement au mouvement d'opposition qui tentait de se former, notamment au sein des universités. Cet ultime épisode a conduit de nouveau à l'arrestation de plusieurs personnes du mouvement dont Rached Ghannouchi qui a été condamné aux travaux forcés à perpétuité.

L'absence de pluralisme politique et l'inexistence de la société civile favorisent la montée des mécontentements populaires qui s'expriment en dehors du champ institutionnel et qui confinent à la contestation du système politique en place:

Dès les années 1970, des forces d'opposition se constituent et pullulent. Des combats éclatent notamment dans la région de Gafsa en 1980, opposant d'une part, les forces du régime à, d'autre part, des rebelles armés, souvent constitués de Tunisiens expatriés et soutenus par des partis étrangers. Cette situation explosive a par ailleurs favorisé le développement des forces islamistes qui organisent à

24. «La Confédération générale des travailleurs tunisiens (CGTT) est un syndicat tunisien créé en 1925 par Mohamed Ali El Hammi. Comme son nom l'indique, son but est de fédérer l'ensemble des syndicats qui émergent à cette époque en Tunisie. C'est un syndicat autonome inédit sous l'empire colonial et qui organise des manifestations et grèves vues d'un très mauvais œil par les autorités coloniales. C'est la raison pour laquelle le protectorat réprimera ce mouvement dont les meneurs et dirigeants seront soit arrêtés soit contraints à s'exiler. Son leader charismatique sera jugé, condamné, expatrié et mourra en 1928, entraînant avec lui cette première expérience syndicale. En 1937, Belgacem Gnaoui fonde la $2^{\mathrm{e}}$ confédération générale des travailleurs tunisiens, qui sera dirigée à partir de 1938 par Hédi Nouira. Elle disparaît en 1940. Après la Seconde Guerre mondiale, des travailleurs tunisiens membres de la CGT française (confédération générale des travailleurs) décident de quitter ce syndicat pour fonder leur formation tunisienne. "C'est alors que, le 19 novembre 1944, furent fondés les syndicats autonomes du Sud, et le 6 mai 1945 , furent fondés les syndicats autonomes du Nord. Quelques mois après, les deux syndicats s'allièrent avec la fédération des fonctionnaires pour former la future Union générale tunisienne du travail (UGTT)"» (W. Tamzini, La Tunisie, p. 13-14).

25. C. Braun, «À quoi servent les partis tunisiens?... », p. 33 .

26. Un surnom courant désignant Habib Bourguiba. 
leur tour des manifestations et des émeutes conduisant la Tunisie à la lisière de la guerre civile. À cela s'ajoute une crise financière qui conduira Bourguiba à limoger, de nouveau, son Premier ministre pour le remplacer par Rachid Sfar et dont la mission essentielle consistera à rétablir l'équilibre économique interne et a assurer une meilleure prospérité du pays. Cette lutte pour le développement économique va très vite laisser place à la lutte contre l'islamisme ${ }^{27}$.

Cette lutte s'intensifiera avec les attentats d'août 1987 et conduira à la destitution du chef de l'État par son Premier ministre en exercice, Zine el-Abidine Ben Ali. Ce n'est qu'après le coup d'État médical du 7 novembre 1987 que le Mouvement de la tendance islamique sortira de sa clandestinité. L'ancien général d'armée devenu notamment Premier ministre durant le dernier mandat d'Habib Bourguiba mettra en place un pacte national appelé Déclaration du 7 novembre et dont les discussions feront participer tous les partis politiques, ainsi que l'ensemble des organisations sociales et syndicales du pays. En ce sens, il décidera de libérer les membres du mouvement qui avaient été emprisonnés sous l'ancien régime et, en contrepartie, le Mouvement de la tendance islamique acceptera d'être rebaptisé Mouvement de la renaissance (al-nahdha) et de soutenir le nouveau président de la République. Mais le nouveau régime ne se limitera pas à intégrer - du moins les premiers mois - l'opposition politique la plus virulente à son encontre. Il multipliera les signes d'ouverture démocratique à destination des Tunisiens mais également des pays voisins.

C'est ainsi que le pacte national affichait l'ambition de démocratiser les institutions en réformant le système politique et en assurant la protection des droits et libertés reconnus dans la Constitution.

\section{Le rôle social des partis politiques}

Ces velléités de démocratisation ne dureront pas très longtemps et le monopartisme, comme sous l'ancien régime, continuera de rythmer la vie partisane tunisienne. En revanche, et contrairement aux années précédentes, la société civile commence à émerger en dehors des institutions, conduisant le régime à créer de nouvelles formes de regroupement pour capter ces énergies citoyennes et, surtout, transformant le parti unique en véritable machine à contrôler la société. Ce verrouillage institutionnel ne suffira pas à contenir les contestations et revendications sociales, qui trouveront à s'exprimer en dehors du circuit institutionnel et sous des formes renouvelées, surtout après la chute du président de la République en 2010 et les changements institutionnels qui en découlèrent.

\section{A. Le Rassemblement démocratique constitutionnel: entre verrouillage institutionnel et médiation sociale}

À peine installé à la tête de l'État, Zine el-Abidine Ben Ali transforme le Parti socialiste destourien en Rassemblement constitutionnel démocratique. Créé officiellement le 27 février 1988, le Rassemblement constitutionnel démocratique s'inscrira dans la lignée des grands partis hégémoniques qu'ont été le Néo-Destour puis le Parti socialiste destourien. Quelques mois plus tard, le régime donnera des signes annonciateurs d'une potentielle démocratisation du système électoral en autorisant officiellement le multipartisme en Tunisie avec l'adoption de la loi organique $\mathrm{n}^{\circ}$ 88-32 du 3 mai 1988 encadrant les partis politiques qui le garantit tout en précisant dans son article 3 qu'un "parti politique ne peut s'appuyer fondamentalement dans ses principes, activités et programme sur une religion, une langue, une race, un sexe ou une région ${ }^{28}$. Au pluralisme politique ainsi consacré s'ajouteront la suppression de la présidence à vie de la République ainsi que la limitation du nombre de mandats (pas plus de trois mandats), autant de réformes s'inscrivant dans une démarche de démocratisation des institutions. D'ailleurs, le nouveau chef de l'État a intitulé son pacte national «le renouveau». Signé, notamment, par tous les partis politiques, ce texte présentait l'ambition de faire travailler ensemble les organisations partisanes en vue de modifier le système politique. Cependant, comme en 1959, cette tentative de démocratisation ne résistera pas à l'épreuve des urnes. Les élections législatives de 1989 donneront une large victoire au Rassemblement constitutionnel démocratique et empêcheront toute formation partisane d'opposition d'obtenir des sièges au Parlement.

Le seul parti politique d'opposition qui réussit à obtenir des résultats satisfaisants est le mouvement Ennahda, qui obtient « une moyenne de $14,5 \%$ au niveau national ${ }^{29}$ et «jusqu'à $30 \%$ des votes ${ }^{30}$ dans certaines circonscriptions, mais qui néanmoins sera privé de tout représentant à l'Assemblée. En plus de ne pas être capable de se réformer et d'accepter le libre jeu électoral en intégrant ses opposants à la vie politique, le Rassemblement constitutionnel démocratique prendra en considération le contexte international pour poursuivre dans sa volonté d'exclure les opposants islamistes de la scène politique. En Algérie éclate la guerre civile dès 1988 et ne rassure pas les élites tunisiennes quant à l'intégration des islamistes au jeu politique national. La peur d'importer cette guerre sur le sol national est prégnante et tenace dans les cercles du pouvoir comme chez une grande partie des citoyens. De plus, la guerre du Golfe qui débute dans les années 1990 constitue également un motif de crainte quant à la place

27. W. Tamzini, La Tunisie, p. 17.

28. Art. 3 de la loi organique $n^{\circ} 88-32$ du 3 mai 1988 organisant les partis politiques, Journal officiel de la République tunisienne, 1988, p. 1.

29. C. Braun, "À quoi servent les partis tunisiens?... », p. 37.

30. Ibid. 
que les islamistes pourraient acquérir dans la vie politique. Ces événements conjugués expliqueront l'exil du leader du mouvement Ennahda - Rached Ghannouchi - et conduiront à l'arrestation puis la condamnation à vie des membres dudit mouvement ${ }^{31}$.

En 1993, une nouvelle loi électorale est promulguée, laquelle est censée donner des gages de démocratisation. La loi organique $n^{\circ}$ 93-118 du 27 décembre 1993 en vue de favoriser le pluripartisme à la Chambre des députés a ainsi pu permettre à 19 députés de l'opposition de siéger au Parlement. Seulement 19 sièges sur les 163 que compte l'Assemblée, le résultat est d'autant plus décevant que la principale force d'opposition est interdite de participation électorale. En effet, le régime s'attache à constituer une opposition de façade de façon à minimiser la place du mouvement Ennahda sur l'échiquier politique. Comme l'écrit une auteure,

La nouvelle loi électorale de 1993 démontre ainsi que Ben Ali a pour stratégie de diviser pour mieux régner, en mettant en place une libéralisation politique minimale qui ne peut qu'affaiblir l'opposition en la laissant s'entredéchirer et en la privant de son adversaire principal: le parti au pouvoir ${ }^{32}$.

Deux années plus tard ont lieu les élections municipales auxquelles participent pour la première fois tous les partis légalisés. Malgré cette participation, les partis d'opposition ne remporteront que 6 sièges sur les 3500 existants. Bien que ce faible score marque officiellement et de façon inédite l'entrée de membres de l'opposition sur le devant de la scène politique, il contribuera néanmoins à participer d'un début de déliquescence de l'opposition puisque Mohammed Moada, le chef d'un des principaux partis, le Mouvement des démocrates socialistes, modifiera son attitude vis-à-vis du régime et dénoncera son hégémonie ainsi que l'absence de réelle démocratie. La réponse du président de la République - qui est également président du parti hégémonique - ne se fera pas attendre: Mohamed Moada sera arrêté puis condamné à onze ans de prison pour avoir entretenu des relations avec un pays étranger - la Lybie - et sera ainsi évincé de la scène politique.

Pour la première fois, Ben Ali fait arrêter un député, représentant majeur de l'opposition non islamiste qui a - l'un des premiers - soutenu le président dès son arrivée à la tête de l'État tunisien, comme sa candidature aux élections présidentielles de $1994^{33}$.

Le verrouillage se poursuivra avec l'adoption de la loi constitutionnelle no 99-52 du 30 juin 1999 qui autorise le pluralisme aux élections présidentielles mais qui, là encore, comporte des dispositions très restrictives comme celle imposant aux personnes souhaitant se présenter d'être chef de parti depuis au moins une durée de cinq années. De plus, le parti en question devra avoir des représentants à la Chambre des députés ${ }^{34}$. C'est donc sans surprise que le président de la République Ben Ali sera réélu avec plus de $99 \%$ des suffrages exprimés. Le même scénario se reproduira lors des élections présidentielles de 2004 puis de $2009^{35}$.

Le Rassemblement constitutionnel démocratique remporte également toutes les autres élections. En 2010, un tunisien sur quatre était membre du Rassemblement. Ce mouvement était bien plus qu'un simple parti politique, il était devenu une instance de surveillance généralisée des citoyens puisqu'il était présent partout, même dans des endroits où n'existait pas l'administration. Disposant de relais sur l'ensemble du pays, le mouvement était également devenu une instance de médiation sociale ${ }^{36}$. C'est ainsi que de nombreuses personnes

[...] ont profité de ce système à travers les programmes sociaux, les caravanes sanitaires, les dons de l'Aïd, l'obtention d'autorisations (permis de taxi, permis de construire, ouverture de commerce, de restaurant, de bar, «licence» pour l'organisation de voyages marchands - i.e. la contrebande - et facilitations pour négocier avec les agents d'autorité... bref, toute activité légale ou illégale), l'acquisition d'agréments et la facilitation de démarches auprès de l'administration, l'octroi de statuts ouvrant la voie à des aides ${ }^{37}$.

De même que le parti aidait de nombreux citoyens, il contribuait dans le même temps à l'ascension socioprofessionnelle de ses membres les plus actifs : «Ainsi, en cinq ou six années, un président de cellule de quartier pouvait se notabiliser et se construire une maison de standing ou ouvrir un commerce rentable $»^{38}$.

Mais ce rôle d'entremetteur, construit sur un socle institutionnel et déployé dans la sphère sociale, ne suffira pas à éteindre toute contestation. Bien au contraire, le verrouillage du système politique va conduire les opposants exclus du champ partisan à se regrouper sous forme d'associations ou de mouvements citoyens. Cette tendance explosera après la révolution de 2010, qui verra le nombre de ces actions se multiplier en dehors de tout champ politique et malgré la multiplication du nombre de formations partisanes ainsi que la consécration de la liberté d'expression.

31. C. Braun, «À quoi servent les partis tunisiens ?...», p. 37

32. Ibid., p. 38 .

33. Ibid., p. 41.

34. Ibid., p. 42

35. «Les organisations des droits de l'Homme dénoncent des atteintes aux droits civiques, aux libertés individuelles et collectives. Pour autant Zine Ben Ali ne cesse de se faire réélire en remportant lors de chaque scrutin plus de $93 \%$ des voix. Il ira jusqu'à modifier la Constitution par référendum en 2004 pour supprimer la limitation du cumul des mandats présidentiels et étendre la limite d'âge d'éligibilité de 65 à 75 ans, comme Bourguiba avait fait modifier la même Constitution pour se rendre Président à vie. Cette réforme constitutionnelle a permis à Zine Ben Ali de se représenter en 2009 et était censée lui permettre de garder le pouvoir jusqu'au moins 2014" (W. Tamzini, La Tunisie, p. 19).

36. Voir B. Hibou, «Tunisie. Économie politique et morale d'un mouvement social», Politique africaine, n ${ }^{\circ} 121,2011$, p. 15.

37. Ibid.

38. Ibid. 


\section{B. Le paradoxe de la révolution de jasmin: entre un pluripartisme effectif et la multiplication des mouvements sociaux à l'extérieur du champ partisan}

Les mouvements de contestation ont débuté bien avant 2010 comme l'atteste par exemple la création du Conseil national pour les libertés en Tunisie en 1998. Regroupant des figures connues de la contestation, comme le journaliste Taoufik Ben Brick ou le médecin Moncef Marzouki qui deviendra le premier président de la République tunisienne après la révolution, ce conseil dénonce les atteintes commises par le régime aux libertés individuelles et publiques ainsi que l'absence de pluralisme politique. Mais les membres dudit conseil étaient persécutés et subissaient le sort qui était infligé à tous ceux qui osaient, sans y avoir été autorisés, exprimer leur désaccord ou qui avaient présenté des revendications qui n'auraient pas été contrôlées au préalable. Il a donc fallu que se produise la révolution de 2010 et la chute du régime de Ben Ali pour que puissent s'exprimer plus ou moins librement les contestations politico-sociales, soit par le biais des formations partisanes, soit par le biais de mouvements nouveaux regroupés la plupart du temps sous forme d'association.

L'un des grands apports juridiques de la révolution a été l'adoption dès 2011 d'un texte libéralisant la création de partis politiques. En effet, le décret-loi no 2011-87 du 24 septembre 2011 portant organisation des partis politiques consacre dans son premier article la liberté de constitution des partis politiques ${ }^{39}$. L'article 5 précise qu'il est «interdit aux autorités publiques d'entraver ou de ralentir l'activité des partis politiques de manière directe ou indirecte $»^{40}$. Ces dispositions contrastent clairement avec le régime juridique antérieur encadrant la création de partis politiques. Cela explique la profusion de formations partisanes depuis 2011 puisqu'il existe aujourd'hui plus de deux cents partis politiques en Tunisie. Mais en réalité seuls quelques-uns dominent la vie politique nationale. Les derniers résultats électoraux le démontrent: lors des premières élections municipales libres qui ont eu lieu le 6 mai 2018, deux grandes formations sont arrivées en tête - celles-là même qui sont également majoritaires à l'Assemblée des représentants du peuple - et sont d'une part le mouvement islamiste Ennahda et, d'autre part, le mouvement Nidaa Tounes auquel appartient l'actuel président de la République Béji Caïd Essebsi. Surtout, le dernier scrutin a fait état de près de $33 \%$ de taux de participation. L'abstention est donc le grand vainqueur, illustrant ainsi la profonde défiance des citoyens vis-à-vis des partis politiques traditionnels.
Une autre illustration de cette défiance réside dans la multiplication des mouvements citoyens de contestation et/ou de revendication. S'appuyant sur les nouveaux droits et libertés consacrés par la Constitution du 26 janvier 2014, les citoyens vont constituer des groupes ou associations pour demander la mise en œuvre de ces dispositions. Par exemple, le $1^{\text {er }}$ juin 2017, quatre jeunes ont été arrêtés dans un jardin public à Tunis pour avoir mangé en pleine journée «de manière ostentatoire». Ils ont été condamnés à un mois de prison ferme pour «atteinte à la morale» et «outrage public à la pudeur». Ce n'est évidemment pas la première fois que des individus sont ainsi "pourchassés» en raison de leur non-respect du jeûne ${ }^{41}$. Mais en 2017 un mouvement citoyen s'est formé et une première manifestation a eu lieu le dimanche 11 juin, réclamant le droit de ne pas jeûner pendant le mois sacré. Ce mouvement est mené notamment par des citoyens très actifs sur les réseaux sociaux et rassemble des jeûneurs comme des non-jeûneurs qui considèrent que le ramadan ne doit pas être «contre notre volonté» ou, en arabe, "Mouch Bessif» (qui est par ailleurs le nom du collectif). Ils réclament en outre le respect de l'article 6 de la Constitution qui, selon eux, garantit leur liberté de ne pas jeûner. Dans le même sens, la création de la première association de libres-penseurs atteste d'une volonté citoyenne de faire respecter, notamment, la liberté de conscience. Nous pouvons citer également l'initiative citoyenne Shams ( «Soleil») qui défend les minorités sexuelles en Tunisie et qui a vu le jour en 2015.

Plus récemment, les manifestations de janvier 2018 rassemblées sous la bannière Fech Nestanew ( $Q$ Qu'est-ce qu'on attend») démontrent, et cela malgré une situation sociale et économique difficile pour une majorité de citoyens, que la liberté de parole, d'expression, de manifestation constituent une avancée considérable des acquis fondamentaux. Il y a eu, hélas, la mort d'un manifestant et une sévère répression (avec pas moins de neuf cents personnes arrêtées). Néanmoins, ce genre de manifestations était tout simplement inimaginable avant la révolution de jasmin.

Le rôle des partis politiques en Tunisie a longtemps été confiné à un jeu d'acteurs, dont le scénario était directement écrit par le chef du parti qui était, jusque 2010, également systématiquement le chef de l'État. Cette confusion entre État et parti a pour origine la conception très personnelle que développait Habib Bourguiba à propos du pouvoir et du rapport de ce dernier à la patrie. Le coup d'État médical de 1987 ayant permis à Zine el-Abidine Ben Ali de prendre la tête du régime n'a rien changé à l'hégémonie du parti quasi unique. Bien au contraire,

39. «Ce décret-loi garantit la liberté de constituer des partis politiques, d’y adhérer et d'y exercer des activités. Il a pour objectif de consacrer la liberté de s'organiser politiquement, appuyer et promouvoir le pluralisme politique et de consolider le principe de transparence dans la gestion des partis politiques» (décret-loi $\mathrm{n}^{\circ}$ 2011-87 du 24 septembre 2011 portant organisation des partis politiques, Journal officiel de la République tunisienne, $\mathrm{n}^{\circ} 74$, p. 1973).

40. Ibid.

41. W. Tamzini, «"Tu ne dé-jeûneras point!”. Quelques propos relatifs à la Constitution tunisienne et à la répression des jeûneurs», Droit cri-TIC, 7 juillet 2015, en ligne: http://www.koubi.fr/spip.php?article954. 
quelques mois après son accession au pouvoir et après une timide tentative de démocratisation de la scène électorale, il poursuivait l'œuvre de son prédécesseur et allait plus loin dans le verrouillage institutionnel puisqu'il a permis de mettre en place une opposition légale, avec des partis autorisés, le tout sous contrôle direct du Rassemblement constitutionnel démocratique, parti succédant au Parti socialiste destourien. Ce n'est qu'à partir de 2011 et l'adoption du décret-loi $n^{\circ}$ 2011-87 du 24 septembre 2011 portant organisation des partis politiques que sera consacrée à l'article premier la liberté de constitution des partis politiques ${ }^{42}$.
Malgré la prolifération des partis politiques, des mouvements citoyens voient le jour pour exprimer des demandes sociales et politiques, délaissant ainsi l'option partisane pourtant rendue largement possible depuis la révolution. Si le rôle institutionnel des partis politiques semble être enfin reconnu par les institutions comme l'attestent les discussions actuelles qui ont trait à la modification de la réglementation relative aux partis politiques, il conviendrait de s'interroger sur leur efficacité sur le plan social et leur capacité à ne pas être déconnectés des aspirations populaires comme cela s'est encore produit lors des manifestations de janvier 2018. 